\title{
The attenuation of oscillatory thermo- capillary convection in the oxide melt by a transverse magnetic field
}

\author{
JIN WeiQing ${ }^{\dagger}$, AI Fei, HONG Yong, LUO HaoSu, LIU Yan \& PAN XiuHong \\ Shanghai Institute of Ceramics, Chinese Academy of Sciences, Shanghai 200050, China
}

The effect of a transverse magnetic field on the oscillatory thermocapillary convection in the $\mathrm{NaBi}\left(\mathrm{WO}_{4}\right)_{2}$ melt was studied by using the in-situ observation system. The oscillation was attenuated when the $60 \mathrm{mT}$ magnetic field was applied, as shown by the decrease in the amplitude and the frequency. Furthermore, the oscillation under smaller temperature difference was stabilized after the magnetic field was applied. The magnetic effect could be due to the Lorentz force generated by the interaction between motional ions and the vertical magnetic field. The ionic conductivities were measured to demonstrate the effect of the magnetic field. The solid ionic electrical conductivity increases with the temperature rise, and the melt ionic electrical conductivity was measured to be about $2.0 \times 10^{-4} \Omega^{-1} \cdot \mathrm{cm}^{-1}$. Experimental results manifest that the effect of the magnetic field on anions and cations in the melt makes the flow change to the direction normal to the applied field, so the flow is more orderly and the oscillation is suppressed.

oscillatory thermocapillary convection, high-temperature melt, in-situ observation, magnetic field

\section{Introduction}

It is well known that externally generated magnetic fields may be used to damp the time dependent convective flows present in melts during the growth of semiconductors for obtaining high quality crystals. However, little is known about the kinds of effects that are produced in an oxide melt when a magnetic field is applied. The reason is that the electrical conductivity in an oxide melt is several orders of magnitude smaller than that in a semiconductor melt, so it may be expected that the flow-attenuation effect is negligible in an oxide melt. However, Miyazawa ${ }^{[1]}$ and coworkers observed bulk flow changed when $800 \mathrm{mT}$ magnetic field was applied in $\mathrm{LiNbO}_{3}$ melt. So far as we know, no report concerning the magnetic effect on the thermocapillary convection in oxide melt

Received February 12, 2007; accepted April 16, 2007 doi: $10.1007 / \mathrm{s} 11433-007-0049-3$

${ }^{\dagger}$ Corresponding author (email: wqjin@sunm.shenc.ac.cn)

Supported by the National Natural Science Foundation of China (Grant No. 50331040) and the Innovation Funds from Shanghai Institute of

Ceramics, Chinese Academy of Sciences (Grant No. SCX0623) 
has been made.

This paper is restricted to a consideration of the effect of the magnetic field on the oscillatory thermocapillary flow in the high temperature oxide melt. Surface tension gradient on the free surface in the melt will drive a flow along the gradient direction, i.e. the thermocapillary convection. In particular, researchers have great interest in the oscillatory thermocapillary convection for its direct connection with the impurity striations ${ }^{[2-8]}$. In our pioneering work ${ }^{[9]}$, the experiments of the thermocapillary flow in high temperature $\mathrm{NaBi}\left(\mathrm{WO}_{4}\right)_{2}$ melt have been carried out. The flow pattern of the steady and oscillatory convection has been visualized. In this study, we mainly discuss the effects of a transverse magnetic field on the oscillatory thermocapillary flow in the oxide melt. Furthermore, the ionic conductivity is measured to interpret the mechanism of the magnetic effect.

\section{Experiments}

Schlieren technique coupled with differential interference microscope has been applied to visualizing the whole process of the flow, as explained elsewhere ${ }^{[10]}$. A short description is given below. The crystal growth unit was made up of a heating chamber and a loop-shaped Pt wire heater, as shown in Figure 1. The Pt wire $(\phi 0.2 \mathrm{~mm})$ loop $(\phi 2.0 \mathrm{~mm})$ was employed to heat and suspend the melt during the experiments. A Pt- $10 \% \mathrm{Rh}$ thermocouple $(\phi 0.08 \mathrm{~mm})$ was used to measure the temperature of the loop. A transverse magnetic field generator was built up to apply the magnetic field parallel to the oxide melt, and the magnetic direction is shown in Figure 2. The final specification of the magnetic field may be controlled from 0 to $140 \mathrm{mT}$.

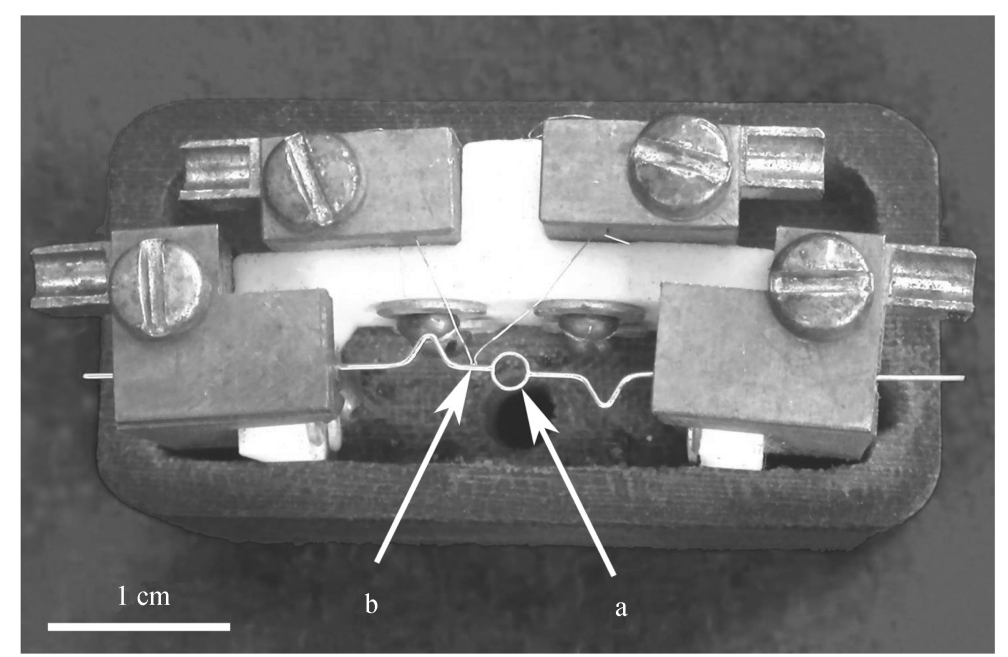

Figure 1 Photo of crystal growth unit. a, Loop-shaped heater; b, thermocouple.

A typical experimental procedure was carried out as follows. The amount of the test melt was precisely controlled until a thin translucent film about $70 \mu \mathrm{m}$ thick was obtained. The temperature distribution in the horizontal direction of the thin melt film was measured by a non-contact $\operatorname{method}^{[11]}$, as shown in Figure 3. The experiments were performed with the melt of $\mathrm{NaBi}\left(\mathrm{WO}_{4}\right)_{2}$, whose melting point is $923^{\circ} \mathrm{C}$, density $7.588 \mathrm{~g} / \mathrm{cm}^{3}$. The magnetic field was applied after the oscillation was triggered, and the change of the flow pattern was observed when adjusting the 


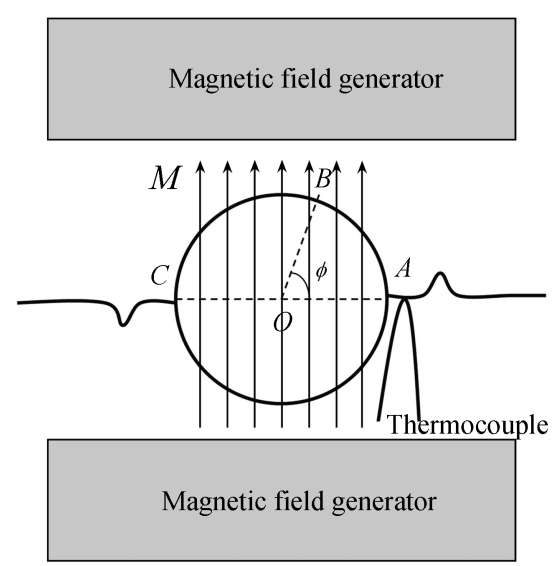

Figure 2 The schematic diagram of the loopshaped heater and the transverse magnetic field. ( $M$ represents the direction of the magnetic field.)

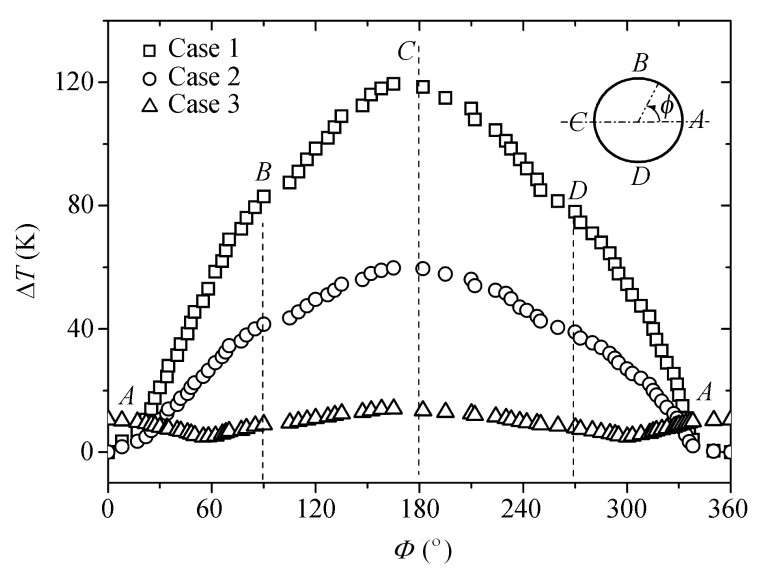

Figure 3 Temperature difference along the azimuthal coordinate of the loop-shaped heater. (Temperature differences between $A$ and $C$ of case 1, 2, 3 are $120,60,10 \mathrm{~K}$, respectively.)

magnetic field.

Solid ionic conductivity measurements were performed on the slice of $\mathrm{NaBi}\left(\mathrm{WO}_{4}\right)_{2}$ crystal $(10$ $\mathrm{mm} \times 10 \mathrm{~mm} \times 1 \mathrm{~mm}$ ). The gilded flat surfaces were used as the electrode for measurement. The samples were equilibrated for about $1 \mathrm{~h}$ prior to each measurement. Melt ionic conductivity experiments were carried out by using the Pt crucible $(10 \mathrm{~mm} \times 20 \mathrm{~mm} \times 20 \mathrm{~mm})$ and two Pt electrode. The resistance was measured by using Keithley 2001 multimeter.

\section{Results and discussion}

A typical flow pattern of $\mathrm{NaBi}\left(\mathrm{WO}_{4}\right)_{2}$ melt comprises one main trunk and branches, as shown in Figure 4(a). In a steady convection stage, the convective flow is symmetric with respect to line $A C$ as shown in Figure 4(b), which is related with the temperature distribution in Figure 3. The temperature difference along the loop generates a surface tension gradient which drives the thermocapillary convection from position $C$ to $A$. When the temperature difference exceeds some critical value, the oscillation of the main trunk is triggered, and arrows I, II represent the range of oscillation as shown in Figure 5(a). Figure 5(b) shows the schematic diagram of the oscillatory pattern. After the oscillations are triggered, the main trunk oscillates around position $A$ with the amplitude shown by the bi-directional arrow 1 . The oscillatory frequency reaches about $10 \mathrm{~Hz}$, and the am-
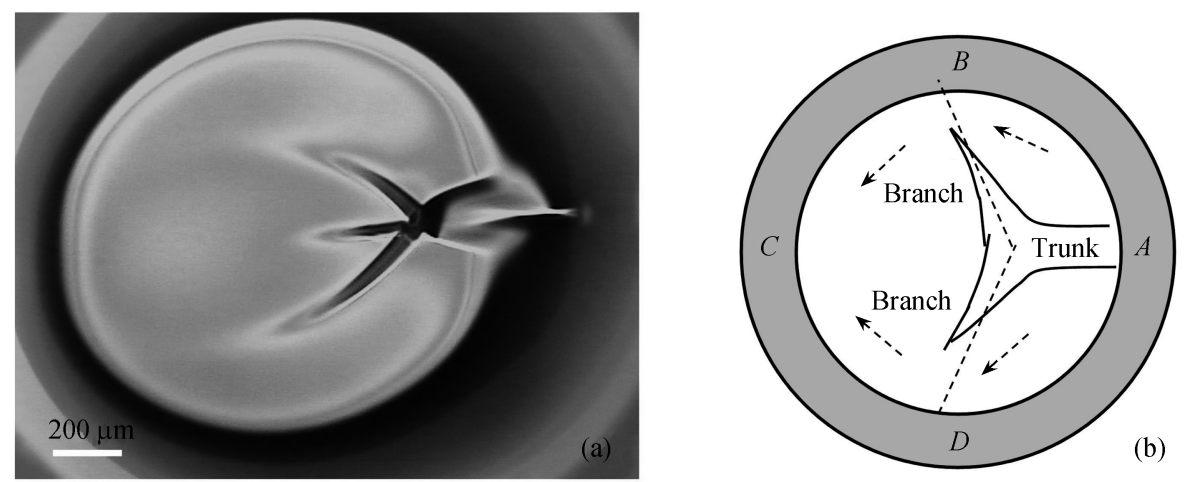

Figure 4 (a) The steady thermocapillary flow pattern; (b) the schematic diagram of the flow pattern. 

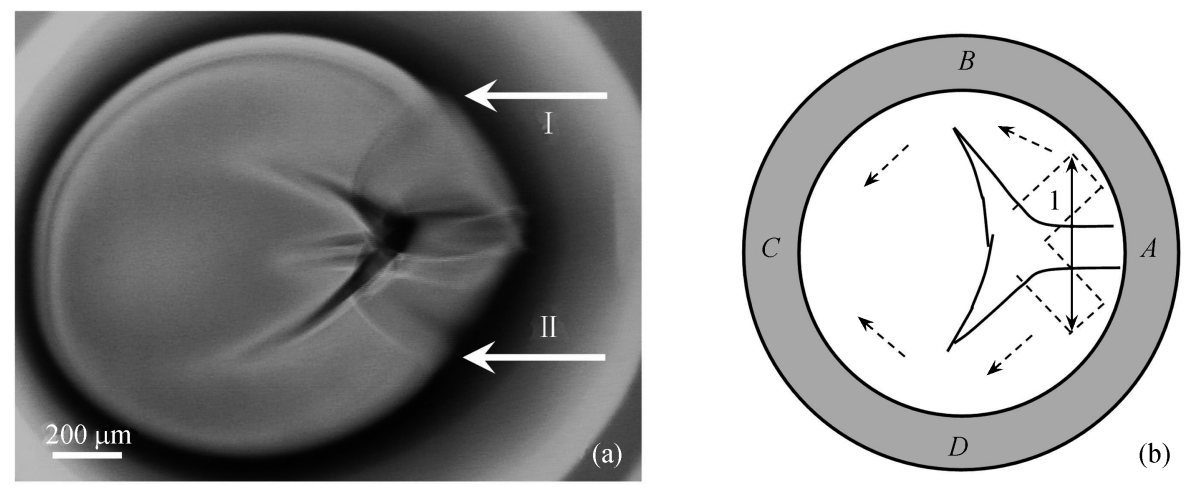

Figure 5 (a) The oscillatory thermocapillary flow pattern; (b) the schematic diagram of the flow pattern.

plitude is about $500 \mu \mathrm{m}$.

When the $60 \mathrm{mT}$ magnetic field is applied, the distinct attenuation of the oscillation is observed and arrows III, IV represent the range of oscillation as shown in Figure 6(a). The main trunk oscillates around position $A$ with the amplitude $\sim 200 \mu \mathrm{m}$, shown by the bi-directional arrow 2 in Figure $6(\mathrm{~b})$, which is smaller than that shown by arrow 1 . So the oscillatory amplitude of the main trunk decreases when the magnetic field is applied. The frequency of oscillation is measured to be about $4 \mathrm{~Hz}$. We would like to emphasize that the oscillation of the thermocapillary flow could be completely suppressed by the $60 \mathrm{mT}$ magnetic field if the temperature difference applied on the loop decreases. This result is inspiring because the impurity striations of crystal can be hopefully reduced or even avoided if the oscillatory thermocapillary convection has been suppressed. Besides, no evident change could be observed with further increase of the magnetic field. However, when the magnetic field is removed, the oscillation of the thermocapillary convection can be observed again with little hysteresis. It is noted that the oscillatory thermocapillary convection could be attenuated by a kind of force which is caused by the magnetic field.
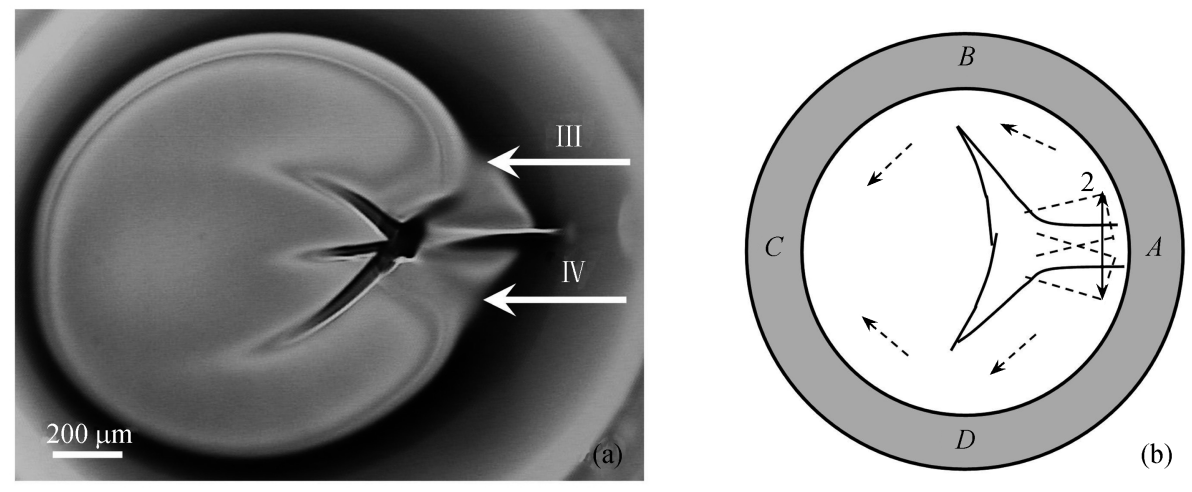

Figure 6 (a) The thermocapillary flow pattern under $60 \mathrm{mT}$ transverse magnetic field; (b) the schematic diagram of the flow pattern.

The magnetic effect must be caused by some kind of thermoelectric effect. The most possible cause is the Lorentz force, which is created by an interaction between the magnetic field and the electric current in the melt. Oxide melts exhibit electric conductivity since they are partially dissociated and exhibit ionic conductivity at high temperature. The motions of ions interact with the 
magnetic field to produce the Lorentz force. The ionic electrical conductivity can be obtained according to the formula $\sigma=(L / A) R$, where $L$ is the thickness of the sample, $A$ the area of the cross section and $R$ the measured resistance. The relation between the ionic conductivity and the temperature obeys the Arrhenius relation, $\sigma=\sigma_{0} \exp (-E / k T)$. Figure 7 shows the change plot of conductivity against temperature. It is obvious that the solid ionic conductivity increases with the rise of temperature, and the melt ionic conductivity is measured to be about $2.0 \times 10^{-4} \Omega^{-1} \cdot \mathrm{cm}^{-1}$. Experimental results manifest that the oxide melts exhibit electric conductivity and the interaction between the magnetic field and the dissociated charged particles, anions and cations creates the Lorentz force, which changes the flow to the direction normal to the applied magnetic field, so the flow pattern is more orderly and the oscillation is suppressed. Further efforts should be made to interpret the mechanism of the magnetic effect.

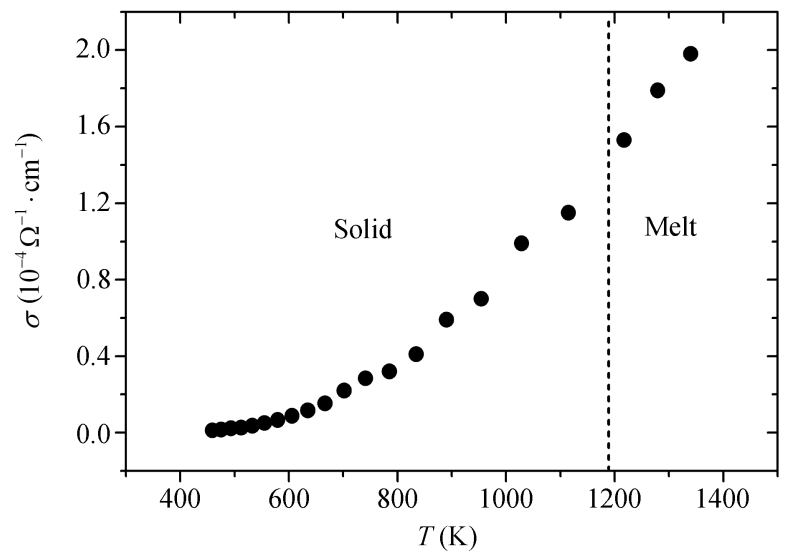

Figure 7 Change of conductivity against temperature.

\section{Conclusions}

The oscillatory thermocapillary convection in high temperature $\mathrm{NaBi}\left(\mathrm{WO}_{4}\right)_{2}$ oxide melt was obviously attenuated by the magnetic field. It was found that the oscillation frequency of the main trunk decreased from $10 \mathrm{~Hz}$ to $4 \mathrm{~Hz}$ when the $60 \mathrm{mT}$ transverse magnetic field was applied. The amplitude of the oscillation also decreased. Moreover, the oscillation in smaller temperature difference could be thoroughly suppressed. Lorentz force generated by the interaction between motional ions and the magnetic field was deemed as the main reason of the attenuation. The ionic electrical conductivity was measured to interpret the mechanism of the magnetic field effect. The solid ionic conductivity increased with the temperature rise and the melt ionic conductivity was turned out to be $2.0 \times 10^{-4} \Omega^{-1} \cdot \mathrm{cm}^{-1}$. It is anticipated that the magnetic field would be reckoned as the effective means to actively control the heat and mass transfer, especially those caused by the thermocapillary flow, in the melt crystal growth industry, although more thorough research work would be required.

1 Miyazawa Y. Flow in oxide melts in a high magnetic field. J Cryst Growth, 1996, 166: 286 -290

2 Schwabe D, Scharmann A. Marangoni convection in open boat and crucible. J Cryst Growth, 1981, 52: 435-449

3 Schwabe D, Velten R, Scharmann A. The instability of surface tension driven flow in models for floating zones under normal 
and reduced gravity. J Cryst Growth, 1990, 99: 1258-1262

4 Tang Z M, Hu W R. Influence of aspect ratio on the onset of thermocapillary oscillatory convection in a floating half zone of large Prandtl number fluid. Chin Phys Lett, 2003, 20: 526-528

5 Jin C J, Hayashi A, Kobayashi M, et al. Effect of internal radiative heat transfer on spoke pattern on oxide melt surface in Czochralski crystal growth. J Cryst Growth, 2003, 259: 367-373

6 Liu Q S, Zhou B H, Nguyen T H, et al. Instability of two-layer Rayleigh-Bénard convection with interfacial thermocapillary effect. Chin Phys Lett, 2004, 21: 686-688

7 Hong Y, Jin W Q, Pan X H. Effect of free surface deformation on thermocapillary convection in high Prandtl number melt. J Cryst Growth, 2005, 274: 480-485

8 Hong Y, Jin W Q, Pan X H, et al. Experimental study on the free surface deformation in the oxide melt. Sci China Ser E-Eng Mater Sci, 2005, 48(5): 481-488

9 Hong Y, Jin W Q, Pan X H. Thermalcapillary convection in NaBi $\left(\mathrm{WO}_{4}\right)_{2}$ melt. Chin Phys Lett, 2004, 21: 1986-1988

10 Jin W Q, Liu Z H, Pan Z L, et al. High temperature in situ observation technique for space experiments. Microgravity Sci Tech, 1997, 4: 194-196

11 Liang X A, Jin W Q, Pan Z L. Experimental measurement of temperature distribution across a loop-like heater. Prog Crystal Growth and Mater Charact, 2000, 40: 301-307 